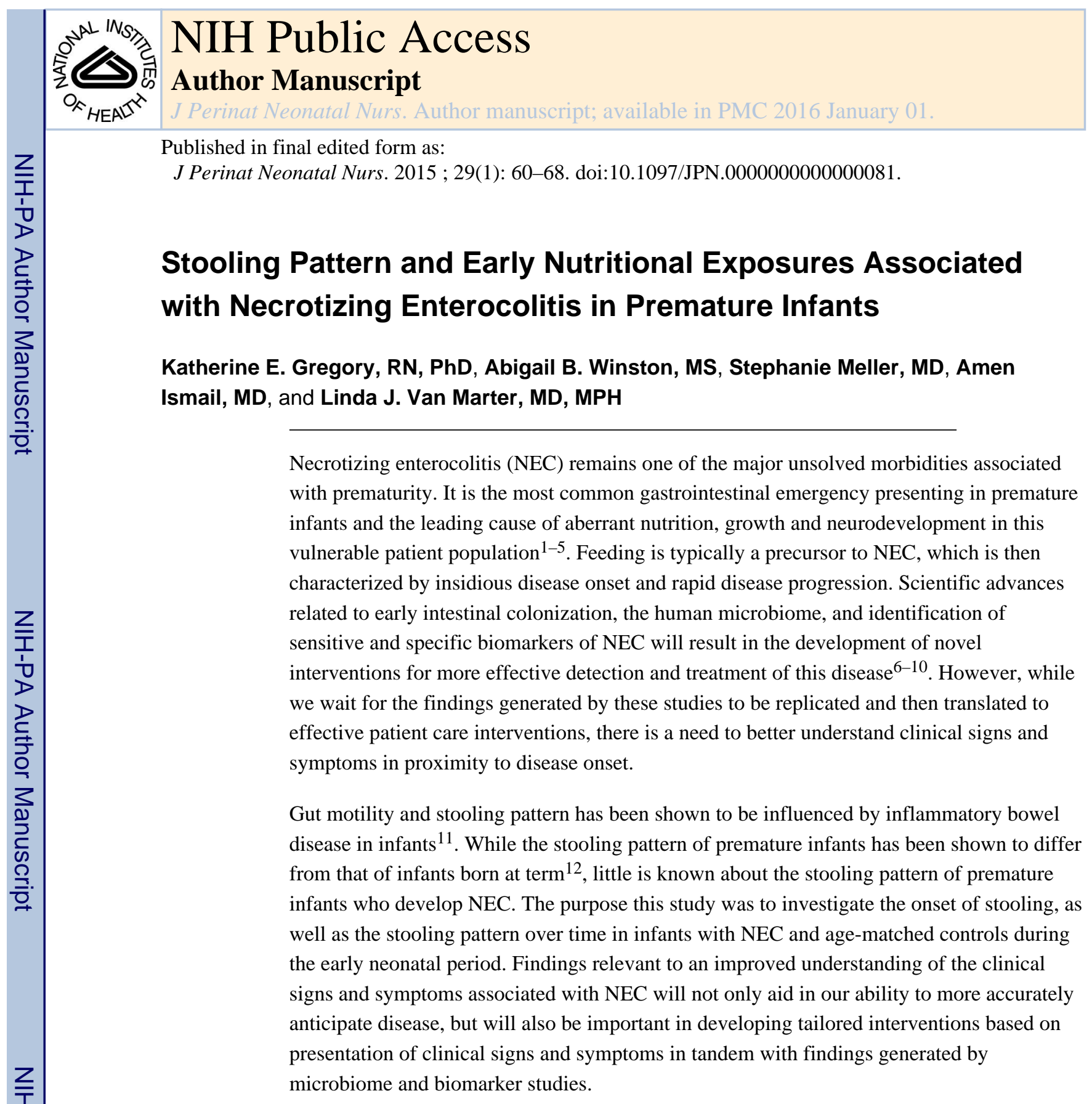

\title{
Background and Significance
}

\section{NEC and Infant Stooling Pattern as a Symptom of Disease}

The only consistently identified clinical risk factor for NEC is prematurity ${ }^{1}$. The premature infant bowel is prone to NEC as a result of aberrant intestinal colonization, inadequate barrier function, poor circulation, an immature immune response, as well as hypomotility $1,13,14$. These factors not only predispose the premature bowel to inflammatory disease, but are likely to result in a stooling pattern that is unique from that of infants born at

\footnotetext{
"corresponding author: Katherine E. Gregory, RN, PhD, Senior Nurse Scientist, Brigham and Women's Hospital, Department of Pediatric Newborn Medicine, Department of Nursing, 75 Francis Street, Boston, MA 02115, katherine.greogry.2@bc.edu, 617-501-1914 (mobile).

The authors have no conflicts of interest to declare.
} 
term gestation ${ }^{12,15-17}$. Studies have shown that the first passage of meconium is delayed and that the transition from meconium to transitional stools is prolonged in the premature infant $^{12}$. Interestingly, the type of feeding, classification as small for gestational age, and the number of days the infant required respiratory support did not delay the passage of meconium stools in this analysis ${ }^{12}$. Studies have shown that the delayed passage of stool in the premature infant is a function of both illness severity and gestational immaturity ${ }^{17}$. When antenatal exposures including betamethasone and magnesium sulfate were investigated, it was shown that betamethasone exposure resulted in an earlier passage of stool while magnesium sulfate exposure did not affect the time of first stool in premature infants ${ }^{17}$. Finally, a more recent study has shown that the establishment of regular bowel habits, defined as the passage of at least two bowel movements per day ${ }^{15}$, in the preterm infant is inversely correlated to gestational age and to the first passage of meconium ${ }^{18}$. In this analysis, the time of first meconium passage and time to achieve regular bowel movements were not influenced by the initiation of feeding or type of feeding, oxygen therapy, gender, birth weight, mode of delivery, presence of sepsis, or type of ventilator support $^{18}$.

\section{NEC and Human Milk as a Protective Factor of Disease}

Several protective factors for NEC have been explored, but to date, only feeding with human milk has been shown to reduce the risk of this disease in premature infants ${ }^{19-23}$. Human milk is thought to protect against NEC as a result of the potent immunological factors unique to human milk and in turn, the role it plays in ensuring early optimal bacterial colonization of the infant bowel ${ }^{1,19}$. Unfortunately, mother's breast milk is not always available in sufficient quantities to premature infants and as a result, they do not receive the full immunologic and microbiologic benefit from this nutritional exposure. Quantifying the percentage of human milk within the premature infant diet necessary to confer a protective effect against NEC is an important step towards better understanding the unique association between early nutritional exposures and NEC.

While it has been shown that the preterm infant bowel pattern is different from that of the term infant (14-16), and part of the Bell Staging criteria that have been used to differentiate disease severity in NEC cases includes changes in stooling ${ }^{24}$, analysis of the differences in stooling within the preterm infant patient population in the context of a GI disease such as NEC is not well understood. Thus, we conducted this investigation exploring the initiation of stooling, early stooling pattern, and frequency of stooling in proximity to disease onset in a retrospective case-control study involving 258 premature infants born prior to 29 weeks gestational age.

\section{Methods}

\section{Participants and Study Design}

In this retrospective case-control study, we assessed the early nutritional exposures and stooling patterns of 258 infants born prior to 29 weeks of gestation. 129 infants developed NEC and 129 unaffected infants served as controls. Infants were drawn from a larger study (HL 67669) of 1,178 extremely low gestational age infants born between 1997 and 2009 at 
Brigham and Women's Hospital. NEC cases were matched 1:1 to NEC cases by gestational age cohort (less than 27 weeks gestation, and 27 to 29 weeks gestation). Each case was matched with the next infant born who met the gestational age matching criteria and did not develop NEC. Infants who were diagnosed with spontaneous intestinal perforation were not included in this study.

\section{Definition of NEC}

Case group assignment required diagnosis of NEC, as defined by Bell Stages I to III ${ }^{24,25}$. Of the 129 infants who met Bell Stage criteria for NEC diagnosis, classification into Stages I, II, and III was as reported in Table 1. While Bell Stage I, the mildest form of the disease, has often been excluded in studies on NEC, we purposely included infants representative of all disease severities (Bell Stages I, II, and III). This allowed us to develop a better overall understanding of GI symptoms associated with all types of disease severity.

\section{Data Collection and Analysis}

All data were obtained from the medical record following research strategies ensuring valid and reliable retrospective data collection ${ }^{26}$. Demographic data collected includes gestational age at birth, birth weight, and gender. Perinatal data collected includes mode of delivery, Apgar scores, and resuscitation efforts required at time of birth. Neonatal data collected includes use of an umbilical arterial catheter, day enteral feeding was initiated, type and volume of first enteral feeding, type and volume of feeding per day, and number of stools per day. Detailed feeding and stooling data was collected on a daily basis until NEC diagnosis for cases or, for controls, until the day of diagnosis for the matched case, for up to 28 days following birth. All feeding data represented either maternal breast milk or infant formula; donor milk was not yet part of clinical care at the time of this study.

Data up to day of disease diagnosis or, for controls, to the day of the matched case's diagnosis, is included. Medians, percentages, and non-parametric tests of significance were used to assess differences in demographic, perinatal, neonatal, feeding, and stooling characteristics between cases and controls. For continuous and ordinal data, Mann-Whitney $\mathrm{U}$ tests were used to compare the case group to the control group, and when comparing groups by NEC stage, significance was assessed by Kruskal-Wallis tests. For categorical data, Chi-Squared tests were used. Cumulative initial stool by postnatal day was calculated for cases and separately for controls.

Linear Mixed Models with unstructured covariance were used to estimate stooling changes in the week prior to disease diagnosis. Day of NEC diagnosis was modeled as Time $=0$, the day prior Time $=-1$, and so on for days prior to disease. The control group was used for comparison using the diagnosis postnatal day of the matched cases. Differences in slope between case and control groups were estimated. Because our findings showed that infants stool more frequently as they age, we cohorted the infants by their postnatal week of NEC diagnosis and analyzed each of these groups separately. Data was collected only until postnatal day 28. Thus, those infants diagnosed with NEC after their fourth postnatal week could not be included in this analysis. All statistical analyses were performed using SAS software, version 9.3 (SAS Institute Inc.; Cary, NC). 


\section{RESULTS}

Our study population was comprised of $109(42 \%)$ girls and $149(58 \%)$ males. The median birth weight was 810 [680-1000] grams and median gestational age was 26 [24.7-27.3] weeks. The majority of infants were born via cesarean section (70.2\%). Median Apgar scores at one and five minutes were 5 and 7 , respectively. There were no statistically significant differences among NEC cases and controls in the demographic, perinatal, or neonatal data, including feeding data such as postnatal day enteral feeding was initiated, the volume of the first feeding, and type of first feeding (Table 1). Descriptive feeding and stooling data is presented in Table 2 .

\section{Stooling Pattern}

The premature infants included in this study passed their first stool, on average, two days following birth. Infants who were diagnosed with NEC did not differ significantly from controls on the day of first stool or the mean or max number of stools per day after the first week following birth. However, infants who developed medical NEC (Stage I and II) had a greater maximum number of stools per day $(p=0.04)$ and a greater mean number of stools per day $(p=0.02)$ when compared to healthy controls during the first week following birth. In controls, the maximum stools in a day during the first week was 2[1-3] and the mean number of stools per day was 0.6 [0.3-1.0]. The mean stools per day for controls gradually increased over the first four weeks following birth to an average of 2.6 stools per day during week 4. In NEC cases, the mean number of stools per day during the first week following birth ranged from 0.42 [0.14-1.10] in infants with Stage III NEC to 1.00 [0.42-1.60] in infants with Stage II NEC. This difference in the number of stools per day was statistically significant $(p=0.02$ ), but only in the first week following birth. There were no statistically significant differences in the mean or maximum number of stools per day in the second, third, or fourth weeks following birth. This data is presented in Table 2.

Pairwise comparisons were conducted comparing the mean stools per day of each case to their matched control. Anticipating that stooling could change with proximity to NEC diagnosis, we grouped the infants based on the postnatal week of NEC diagnosis and analyzed each group separately. These findings are reported in Table 3 and showed no significant differences between the mean numbers of stools per day in infants who developed NEC and their matched controls.

The cumulative distribution of stooling onset in cases and controls was evaluated. As seen in Figure 1, there were no major differences observed among cases and controls. Most cases $(60 \%)$ and controls $(65 \%)$ passed their first stool within three days following birth and the vast majority of both cases (95\%) and controls (91\%) passed their first stool within the first week following birth. When the stooling pattern was evaluated in proximity to NEC diagnosis, analyses were conducted based on whether or not the infant was diagnosed with NEC during week 1, 2, 3, or 4. Those developing NEC later were not included in this analysis. This data is shown in Figure 2, which illustrates the average stools per day for the days prior to NEC diagnosis, with 0 indicting NEC diagnosis day. For controls, day of diagnosis is based on each control's matched case, as described earlier. Overall, the number of stools in both NEC cases and controls increased as the infant attained greater gestational 
age, but there were no statistically significant differences in the number of stools between NEC cases and controls in the week prior to NEC diagnosis for infants diagnosed in the first three weeks (Figures 2A-2C). For infants diagnosed in week 4, there was a statistically significant but not clinically meaningful increase in the number of stools as the infants approached diagnosis when compared to their matched controls (Figure 2D). On average, in the seven days prior to diagnosis, the cases increased at a rate of 0.02 stools per day while the controls decreased at a rate of 0.07 stools per day $(\mathrm{p}=0.03)$.

\section{Early nutritional exposures: Feeding with maternal breast milk}

The majority of the subjects had received their first feeding by day 5 [3.0-9.0] following birth. Control infants were more frequently fed breast milk at the time of first feed (69.6\%) when compared to infants who developed NEC ( $\mathrm{p}=0.03$ ). When the percentage of feeding with breast milk was compared over time prior to diagnosis with NEC, infants who developed Stage I and II NEC received a lower percentage of their feedings from breast milk ( $85 \%$ and $88 \%$, respectively) when compared to controls $(100 \%)(p=0.02)$.

The number of days infants were not fed (NPO) during the first week following birth differed significantly only among Stage II NEC cases and controls, and only in the first postnatal week. On average, infants in the control group remained NPO for 5 days, while infants who eventually developed Stage II NEC were NPO for 3 days during the first week following birth $(p=0.05)$.

\section{DISCUSSION}

This descriptive analysis of the onset and pattern of stooling among low gestational age infants with and without NEC shows that during the first four weeks following birth, few differences exist in the day of first stooling, maximum and mean number of stools per day, and the number of stools per day in the week prior to disease onset. The differences that were identified primarily occurred in the first week following birth. During this time, infants who ultimately developed Stage II NEC stooled slightly more frequently. This may be explained by the finding that these infants were NPO for slightly fewer days when compared to their controls. However, in the pairwise analysis, when comparing cases to their matched controls and separating groups by week of NEC onset, we did not identify any notable differences in the mean number of stools per day (Table 3). Because this pairwise analysis compared infants to those within their same gestational age cohort, and since NPO is likely influenced by gestational age, perhaps both gestational age and NPO are good candidates for future study of stooling first postnatal week. We did not see any difference in the day of first stooling between cases and controls.

It was interesting to observe that the vast majority of infants passed their first stool in the week following birth. For the small number of infants who did not stool in this time frame, we explored some of the clinical reasons why they might have had delayed motility (i.e. maternal magnesium, GI disease). In these 20 infants, we found that approximately half $(n=9)$ of their mother's received magnesium. We also found that $40 \%(n=8)$ developed NEC on or before postnatal day 5 . This very early onset and treatment for NEC was likely to have contributed to a delay in stooling in these infants. 
In analyzing our stooling data in proximity to disease onset, we found that overall, infants had a greater number of stools each day as they attained postnatal age. This was not surprising and was a similar finding to the work others have reported ${ }^{15}$. What was interesting to note was that overall, there were no major differences in the number of stools between cases and controls as the infants approached disease diagnosis. This was especially true for infants who were diagnosed with NEC two and three weeks following birth. For the infants who were diagnosed during week 1 , controls trended towards a greater number of stools when compared with cases on the same day, but this trend was not significant. For the infants who became ill during week 4, the infants with NEC had a slight increase in the number of stools as they approached disease while the controls were slightly decreasing in number of stools during that week. This change was statistically significant, but again, the overall difference in the number of stools was small, changing by less than a tenth of a stool per day on average for both groups. While these differences were small, it is possible that they may be explained by a differing pathophysiology contributing to NEC that occurs in the very early vs. later neonatal period. It is scientifically plausible that early onset NEC has more to do with fetal biology and a maternal contribution to the disease, while late onset $\mathrm{NEC}$ is the result of neonatal factors such as nutritional and nosocomial exposures in the NICU. These factors may influence the stooling pattern as a result of subtle differences in early intestinal colonization and overall function of the bowel as the infant matures.

Infants who developed NEC were more frequently fed infant formula for their first feeding, as expected given the results of previous studies. A statistically significant difference was also identified in the percentage of feeding with maternal breast milk prior to disease onset $(p=0.02)$. Interestingly, in this study, even infants who developed NEC received a relatively high percentage of maternal breast milk, on average (i.e. 85\% in Bell Stage I, 88\% in Bell Stage II, and $100 \%$ in Bell Stage III). While there was a much larger range in the percentage of maternal breast milk among the cases when compared to the controls, these observations suggest that the percentage of maternal breast milk necessary for protection against NEC may be as high as $90 \%$. Further study that helps to pinpoint the amount of breast milk required to adequately protect against NEC is needed.

In conclusion, the stooling pattern among low gestational age infants with or without NEC has not previously been studied. The findings of our analyses show that increased stooling frequency during the first week may be associated with later disease, although we only detected an increase among babies who developed Stage II NEC, and the increase might be too small to be clinically useful. Overall, feeding, stooling, and other neonatal factors we investigated of infants who develop NEC are similar to those who are unaffected by this GI disease. These findings are relevant to clinical practice in the NICU, where our clinical suspicion for NEC is often heightened by changes in the stooling pattern. While more study may be needed, the results reported here suggest that there are no major differences in the onset of stooling or the stooling frequency in infants who do or do not develop NEC. This was somewhat surprising because NEC is an inflammatory disease of the bowel where GI signs and symptoms would be expected. However, this data suggests otherwise. As a result, concern for NEC should not be focused on GI signs and symptoms alone. In addition, this is important information for researchers aiming to use stool samples to investigate bowel 
disease, inflammatory disease (such as sepsis), or long-term morbidity (such as allergy, asthma, and growth), in premature infants. Investigators should not anticipate NEC to affect their ability to collect stool samples from the majority of premature infants.

\section{References}

1. Neu J, Walker W. Necrotizing Enterocolitis. New England Journal of Medicine. 2011; 364(3):255264. [PubMed: 21247316]

2. Lin P, Stoll B. Necrotizing enterocolitis. The Lancet. 2006; 368(9543):1271-1283.

3. Hintz SR, Kendrick DE, Stoll BJ, et al. Neurodevelopmental and Growth Outcomes of Extremely Low Birth Weight Infants After Necrotizing Enterocolitis. Pediatrics. 2005; 115(3):696-703. [PubMed: 15741374]

4. Schulzke S, Deshpande G, Patole S. Neurodevelopmental outcomes of very low-birth-weight infants with necrotizing enterocolitis: A systematic review of observational studies. Archives of Pediatrics \& Adolescent Medicine. 2007; 161(6):583-590. [PubMed: 17548764]

5. Rees CM, Pierro A, Eaton S. Neurodevelopmental outcomes of neonates with medically and surgically treated necrotizing enterocolitis. Archives of Disease in Childhood - Fetal and Neonatal Edition. 2007; 92(3):F193-F198. [PubMed: 16984980]

6. Mai V, Young CM, Ukhanova M, et al. Fecal microbiota in premature infants prior to necrotizing enterocolitis. PloS one. 2011; 6(6):e20647. [PubMed: 21674011]

7. Torrazza RM, Ukhanova M, Wang X, et al. Intestinal microbial ecology and environmental factors affecting necrotizing enterocolitis. PloS one. 2013; 8(12):e83304. [PubMed: 24386174]

8. Claud EC, Keegan KP, Brulc JM, et al. Bacterial community structure and functional contributions to emergence of health or necrotizing enterocolitis in preterm infants. Microbiome. 2013; 1(1):20. [PubMed: 24450928]

9. Reisinger KW, Van der Zee DC, Brouwers HA, et al. Noninvasive measurement of fecal calprotectin and serum amyloid A combined with intestinal fatty acid-binding protein in necrotizing enterocolitis. Journal of pediatric surgery. 2012; 47(9):1640-1645. [PubMed: 22974599]

10. Gregory KE, Winston AB, Yamamoto HS, et al. Urinary intestinal fatty acid binding protein predicts necrotizing enterocolitis. The Journal of pediatrics. 2014; 164(6):1486-1488. [PubMed: 24636854]

11. Kwon YH, Kim YJ. Pre-diagnostic Clinical Presentations and Medical History Prior to the Diagnosis of Inflammatory Bowel Disease in Children. Pediatric gastroenterology, hepatology \& nutrition. 2013; 16(3):178-184.

12. Bekkali N, Hamers SL, Schipperus MR, et al. Duration of meconium passage in preterm and term infants. Archives of disease in childhood. Fetal and neonatal edition. 2008; 93(5):F376-F379. [PubMed: 18285377]

13. Berseth CL. Gestational evolution of small intestine motility in preterm and term infants. The Journal of pediatrics. 1989; 115(4):646-651. [PubMed: 2507768]

14. Ittmann PI, Amarnath R, Berseth CL. Maturation of antroduodenal motor activity in preterm and term infants. Digestive diseases and sciences. 1992; 37(1):14-19. [PubMed: 1728520]

15. Weaver LT, Lucas A. Development of bowel habit in preterm infants. Archives of Disease in Childhood. 1993; 68(3 Spec No):317-320. [PubMed: 8466270]

16. Verma A, Dhanireddy R. Time of first stool in extremely low birth weight ( $<$ or $=1000$ grams) infants. J Pediatr. 1993; 122(4):626-629. [PubMed: 8463914]

17. Kumar SL, Dhanireddy R. Time of first stool in premature infants: effect of gestational age and illness severity. The Journal of pediatrics. 1995; 127(6):971-974. [PubMed: 8523200]

18. Baldassarre M, Fanelli M, Laneve AM, et al. Bowel habit in preterm newborns: effect of new formulas. Endocrine, metabolic \& immune disorders drug targets. 2010; 10(2):175-178.

19. Walker WA. Breast Milk as the Gold Standard for Protective Nutrients. The Journal of Pediatrics. 2010; 156(S):3-7. [PubMed: 20006756] 
20. Sisk PM, Lovelady CA, Dillard RG, Gruber KJ, O'Shea TM. Early human milk feeding is associated with a lower risk of necrotizing enterocolitis in very low birth weight infants. $\mathrm{J}$ Perinatol. 2007; 27(7):428-433. [PubMed: 17443195]

21. Meinzen-Derr J, Poindexter B, Wrage L, Morrow AL, Stoll B, Donovan EF. Role of human milk in extremely low birth weight infants' risk of necrotizing enterocolitis or death. J Perinatol. 2009; 29(1):57-62. [PubMed: 18716628]

22. Rautava S, Nanthakumar NN, Dubert-Ferrandon A, Lu L, Rautava J, Walker WA. Breast MilkTransforming Growth Factor- $\beta_{2}$ Specifically Attenuates IL-1 $\beta$-Induced Inflammatory Responses in the Immature Human Intestine via an SMAD6- and ERK-Dependent Mechanism. Neonatology. 2011; 99(3):192-201. [PubMed: 20881435]

23. Newburg DS, Walker WA. Protection of the neonate by the innate immune system of developing gut and of human milk. Pediatr Res. 2007; 61(1):2-8. [PubMed: 17211132]

24. Bell M, Ternberg J, Feigin R, et al. Neonatal necrotizing enterocolitis. Therapeutic decisions based upon clinical staging. Annals of Surgery. 1978; 187(1):1-7. [PubMed: 413500]

25. Lee JS, Polin RA. Treatment and prevention of necrotizing enterocolitis. Seminars in Neonatology. 2003; 8:449-459. [PubMed: 15001117]

26. Gregory KE, Radovinsky L. Research strategies that result in optimal data collection from the patient medical record. Applied nursing research : ANR. 2012; 25(2):108-116. [PubMed: 20974093] 

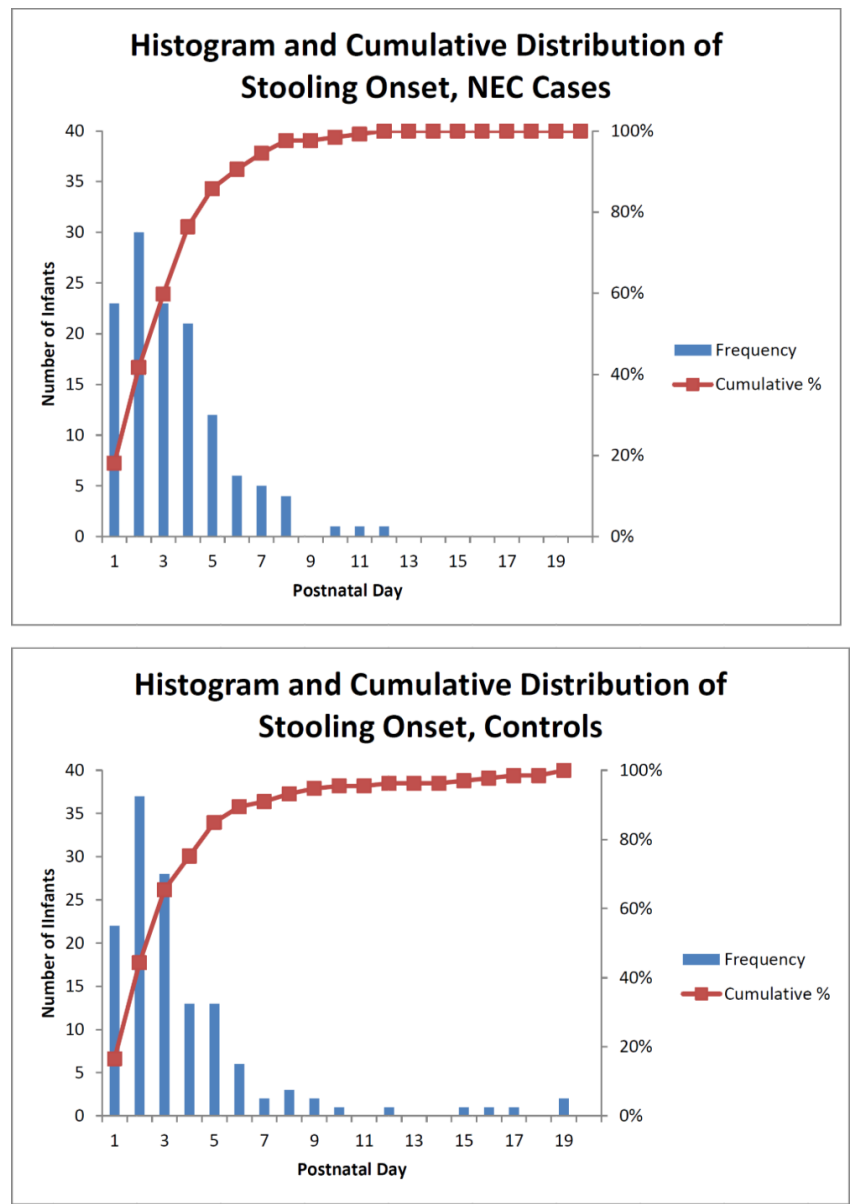

Figure 1.

Distribution of Stooling Onset, NEC Cases and Controls 


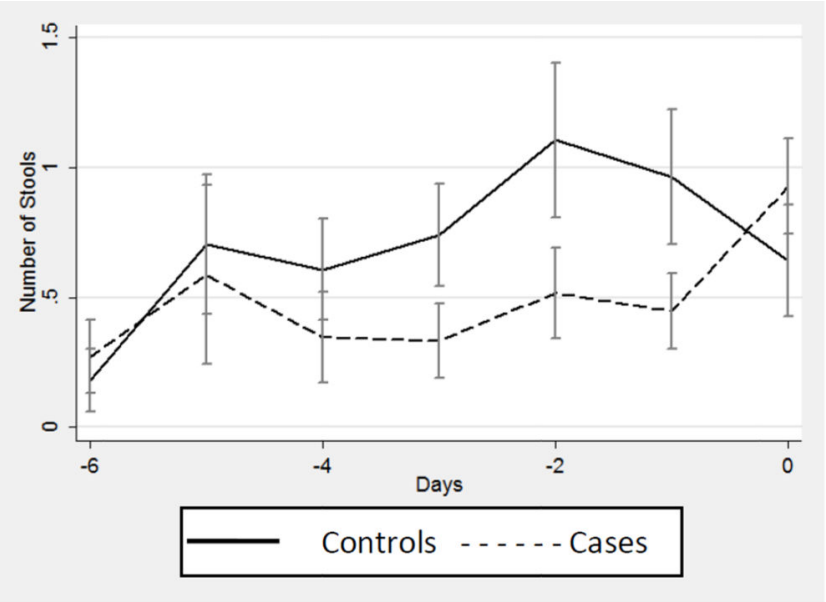

a. Infants who were diagnosed with NEC in week $1(n=129)$

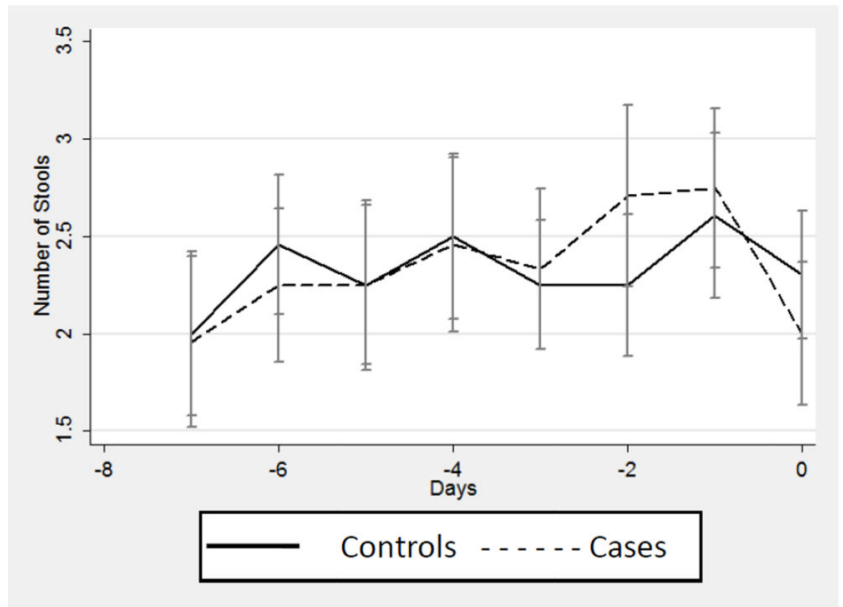

c. Infants who were diagnosed with NEC in week $3(n=60)$

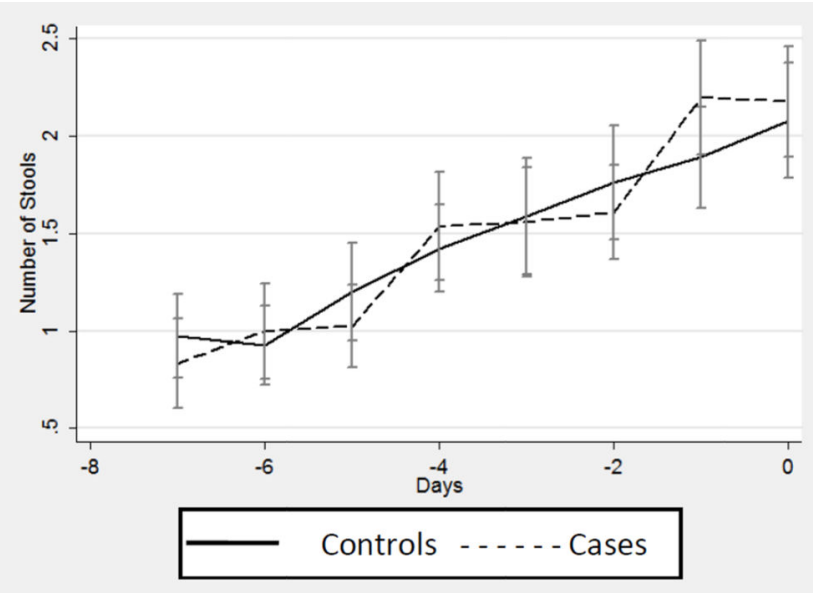

b. Infants who were diagnosed with NEC in week $2(n=99)$

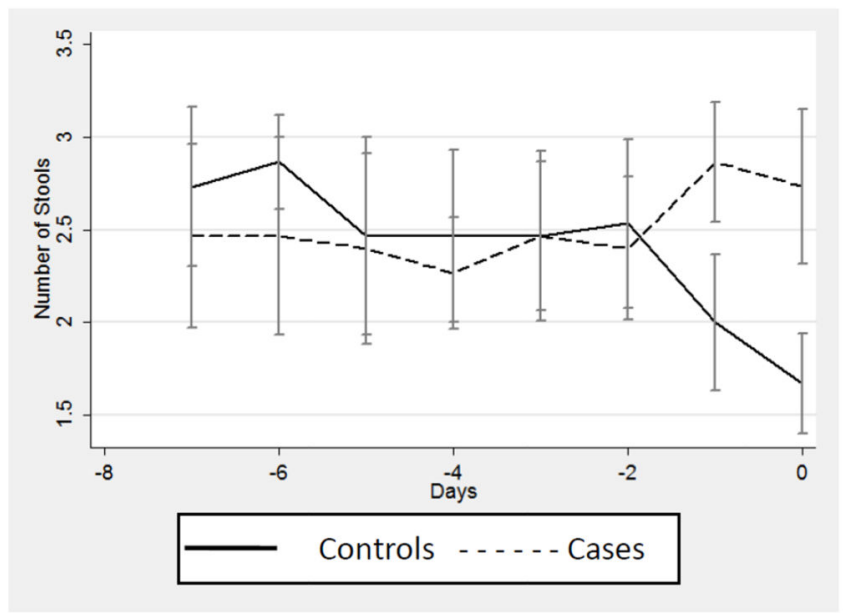

d. Infants who were diagnosed with NEC in week 4 ( $n=39)$

Figure 2.

Stooling pattern in proximity to NEC onset day, as indicated by 0 on $\mathrm{x}$-axis

$\mathrm{x}$-axis: days prior to disease diagnosis day, which is indicated by 0 ; $\mathrm{y}$-axis: number of stools per day 
Table 1

Descriptive demographics, perinatal and neonatal factors

\begin{tabular}{|c|c|c|c|c|}
\hline & $\begin{array}{c}\text { Total Sample } \\
(\mathbf{n}=\mathbf{2 5 8})\end{array}$ & $\begin{array}{c}\text { NEC Cases } \\
(\mathbf{n}=129)\end{array}$ & $\begin{array}{c}\text { Control Subjects } \\
(\mathbf{n = 1 2 9})\end{array}$ & $p$ \\
\hline \multicolumn{5}{|c|}{ Demographics } \\
\hline Gestational Age & $26[24.7-27.3]$ & $26[24.6-27.4]$ & $26.3[25.0-27.3]$ & 0.30 \\
\hline Birth Weight & $810[680-1000]$ & $795[680-1000]$ & $850[700-990]$ & 0.35 \\
\hline \multicolumn{5}{|l|}{ Gender } \\
\hline Female & $109(42.2)$ & $51(39.5)$ & $58(44.9)$ & \\
\hline Male & $149(57.8)$ & $78(60.5)$ & $71(55.1)$ & 0.38 \\
\hline \multicolumn{5}{|c|}{ Perinatal Factors } \\
\hline \multicolumn{5}{|l|}{ Mode of Delivery } \\
\hline Vaginal & $77(29.8)$ & $43(33.3)$ & $34(26.4)$ & 0.22 \\
\hline Cesarean Section & $181(70.2)$ & $86(66.6)$ & 95 (73.6) & \\
\hline Apgar score at $1 "$ & $5[3-7]$ & $5[3-7]$ & $5[3-6]$ & 0.11 \\
\hline Apgar score at 5" & $7[6-8]$ & $7[6-8]$ & $7[6-8]$ & 0.11 \\
\hline \multicolumn{5}{|l|}{ DR Resuscitation: } \\
\hline Chest Compressions & $25(10)$ & $12(9.8)$ & $13(10.2)$ & 0.89 \\
\hline Epinephrine & $14(9.3)$ & $6(4.9)$ & $8(6.3)$ & 0.62 \\
\hline \multicolumn{5}{|c|}{ Neonatal Factors } \\
\hline Umbilical Arterial Catheter (UAC) & $171(67.1)$ & $89(69.5)$ & $82(64.6)$ & 0.39 \\
\hline Day enteral feeding initiated & $5[3.0-9.0]$ & $5[3.0-8.5]$ & $5.5[3.0-9.0]$ & 0.77 \\
\hline Volume of first enteral feeding & $1.3[1.0-1.7]$ & $1.2[1.0-1.7]$ & $1.3[1.0-1.7]$ & 0.96 \\
\hline \multicolumn{5}{|l|}{ Type of first enteral feeding } \\
\hline Breast Milk & $155(60.1)$ & $68(52.7)$ & $87(67.4)$ & 0.03 \\
\hline Other (formula) & $83(32.2)$ & $46(35.6)$ & 37 (28.6) & \\
\hline Not fed/missing & $20(7.7)$ & 15 (11.6) & $5(3.8)$ & \\
\hline
\end{tabular}

Median [IQR] reported for: gestational age, birth weight, Apgar scores, day enteral feeding initiated, volume of first enteral feeding $\mathrm{n}(\%)$ reported for gender, mode of delivery, DR resuscitation, UAC, type of first enteral feeding 


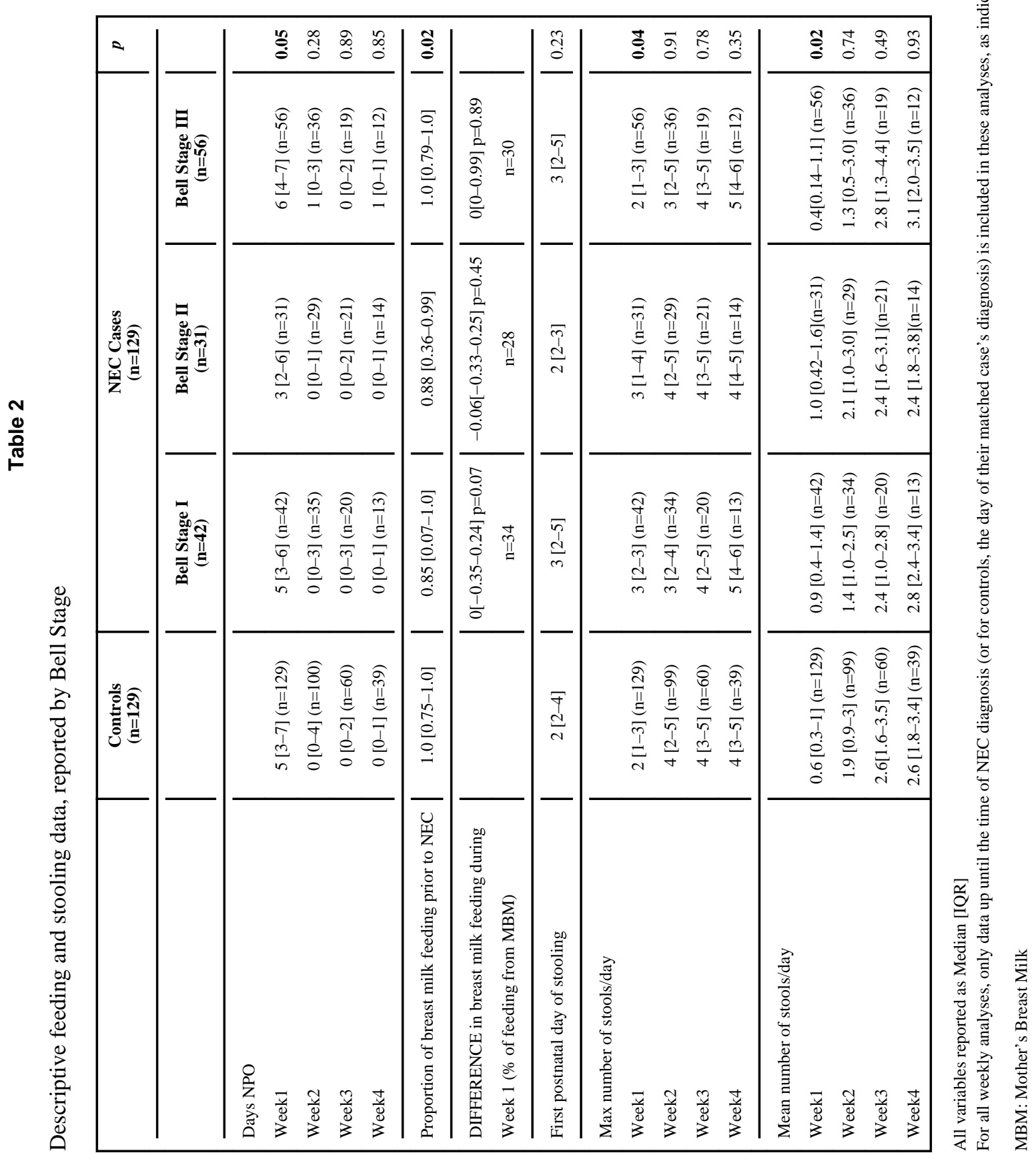

J Perinat Neonatal Nurs. Author manuscript; available in PMC 2016 January 01. 
Table 3

Pairwise comparison of differences between mean number of stools per day, reported by week of NEC onset and by NEC stage

\begin{tabular}{|l|l|l|l|l|}
\hline & $\begin{array}{l}\text { DIFFERENCE in number } \\
\text { of stools/day } \\
\text { Median [IQR] } p\end{array}$ & $\begin{array}{l}\text { Stage I } \\
\text { Median [IQR] } \boldsymbol{p}\end{array}$ & $\begin{array}{l}\text { Stage II } \\
\text { Median [IQR] } \boldsymbol{p}\end{array}$ & $\begin{array}{l}\text { Stage III } \\
\text { Median [IQR] } \boldsymbol{p}\end{array}$ \\
\hline $\begin{array}{l}\text { DIFFERENCE in mean number of } \\
\text { stools/day reported by Bell Stage and Week } \\
\text { of disease diagnosis }\end{array}$ & & & & \\
Week1 (n=129) & $0.0[-0.6,0.7] .38$ & $0.2[-0.3,0.9] .09$ & $0.1[-1.0,1.0] .99$ & $00[-0.6,0.6] .90$ \\
Week2 (n=99) & $0.0[-1.0-1.0] .77$ & $0.0[-1.0,0.5] .30$ & $-0.1[-1.0,1.0] .79$ & $0.1[-1.4,1.9] .47$ \\
Week3 (n=60) & $0.0[-1.1,1.1] .73$ & $0.0[-0.7,1.6] .99$ & $0.1[-2.1,1.0] .35$ & $.21[-1.0,1.0] .90$ \\
Week4 (n=39) & $0.3[-0.7,1.7] .16$ & $0.9[-0.3,2.3] .12$ & $0.1[-1.2,1.7] .89$ & $.21[-0.6,1.0] .48$ \\
\hline
\end{tabular}

